\title{
Spontaneous hexagon formation in photorefractive crystal with a single pump wave
}

\author{
P. M. Lushnikov \\ Landau Institute for Theoretical Physics, Kosygin Street 2, Moscow 117334, Russia
}

A. V. Mamaev

Institute for Problems in Mechanics, Prospekt Vernadskogo 101, Moscow 117526, Russia

Received June 1, 1999

\begin{abstract}
A new scheme for spontaneous formation of hexagonal optical patterns in photorefractive crystals with dominant reflection gratings and a single pump wave is proposed and realized experimentally. It is shown theoretically that spontaneous hexagon formation results from random small-angle scattering of the pump wave inside the crystal. The threshold of spontaneous hexagon formation is found. (c) 1999 Optical Society of America

OCIS codes: $190.0190,190.4420,190.5330$.
\end{abstract}

The interaction of counterpropagating optical waves in nonlinear media often results in transverse instabilities and in the formation of regular spatial structures, mostly with hexagonal symmetry. Hexagonal patterns have been observed in a wide variety of materials, including atomic vapors, liquid crystals, and photorefractive crystals. ${ }^{1}$ Counterpropagating beams interact by means of diffraction on the self-induced reflection or transmission gratings of a nonlinear medium. ${ }^{2,3}$ The threshold of instability and the angular scale of the patterns generated have depended on whether the second pump was produced externally $^{3-6}$ or arose from the first beam by reflection from a feedback mirror placed outside the nonlinear medium. ${ }^{2}$ Two counterpropagating pump waves were essential in all those experiments as well as in theoretical calculations of transverse instabilities. Here we propose, for the first time to our knowledge, and have realized experimentally a new scheme for pattern generation in photorefractive crystals with dominant reflection gratings. The chief unique characteristic of this scheme is the presence of only one pump wave, which, as is shown below, lends unusual properties to the system.

A schematic of the setup is shown in Fig. 1. Coherent laser beam $F_{0}$ is directed into photorefractive crystal C, where recording of reflection gratings of the diffusion type ${ }^{7}$ is possible. The output surface of the crystal is imaged by lenses $L_{1}$ and $L_{2}$, which form a $4 f$ system upon mirror M. Circular slit $\mathrm{D}$ is placed in the common focal plane of these lenses. This circular slit cuts off all angular components of laser radiation except the range $(\theta, \theta+\delta \theta)$, where $\theta<<1$ and $\delta \theta / \theta<<1$. As a result, mirror $\mathrm{M}$ reflects only sideband beams with a transverse wave vectors $\mathbf{k}_{\perp}$ that lie upon ring $\left|\mathbf{k}_{\perp}\right| \simeq \theta k_{0}$, where $k_{0}$ is the wave vector of the pump beam.

We performed the experiment by using the principal configuration shown in Fig. 1. A crystal of $\mathrm{KNbO}_{3}: \mathrm{Fe}$ (0.5 wt.\%) measuring $l=5.2 \mathrm{~mm}$ along the $c$ axis was illuminated by a $35-\mathrm{mW}$ laser beam at $\lambda=632.8 \mathrm{~nm}$ focused onto a spot with a Gaussian diameter of $\sim 0.3 \mathrm{~mm}$. The light was polarized parallel to the crystal $a$ axis to take advantage of the $r_{13}$ electrooptic coefficient. The crystal was slightly tilted $\left(\simeq 10^{\circ}\right)$ to avoid the influence of the beam reflected from the back surface of the crystal. The circular slit cut the pump beam off and passed only a part of the scattered radiation. This scattered light is due to the scattering of the pump on the crystal inhomogeneities and represents a speckle field in the plane of filtering. The width of the circular slit was chosen to coincide approximately with a transverse size of a speckle spot. The crystal was oriented such that the scattered light reflected from mirror $\mathrm{M}$ was amplified by pump $\mathrm{F}_{0}$. A part of the output radiation reflected by a beam splitter (not shown in Fig. 1) was used for recording near- and far-field pictures and the time dependence of the generated radiation.

The experimental results are presented in Figs. 2 and 3. Figure 2 shows images of near-field patterns at the input surface of the crystal (right) and farfield patterns (left) for two values of the circular slit diameter. Note that increasing the width of the circular slit to 3-4 speckle-spot size destroys the hexagonal symmetry of the generated radiation.

Typical temporal dynamics of the generated radiation for two values of the coupling constant are shown in Fig. 3. If the system is below the threshold for spontaneous hexagon formation (curve 1), the power of the generated wave tends to a low plateau determined by

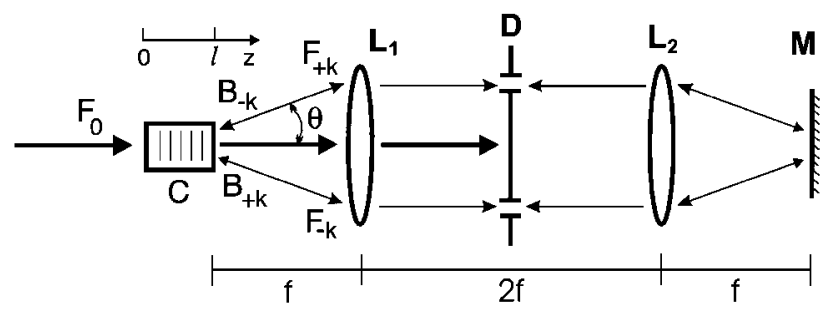

Fig. 1. Schematic of the setup for hexagon formation. Abbreviations are defined in text.

(C) 1999 Optical Society of America 

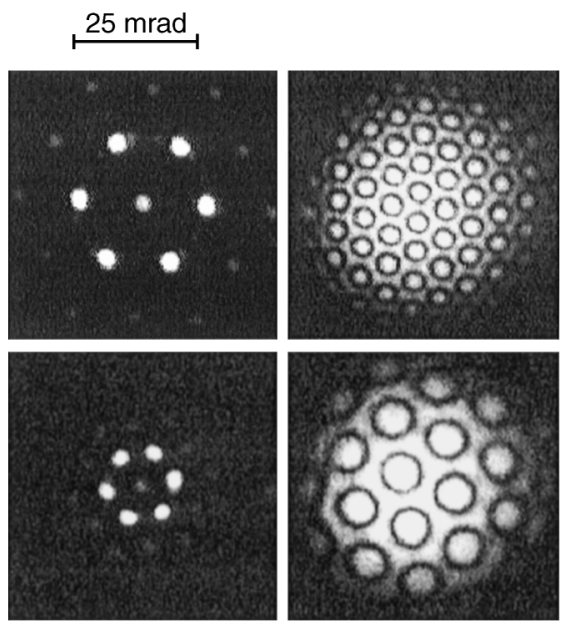

Fig. 2. Near- and far-field images of generated light.

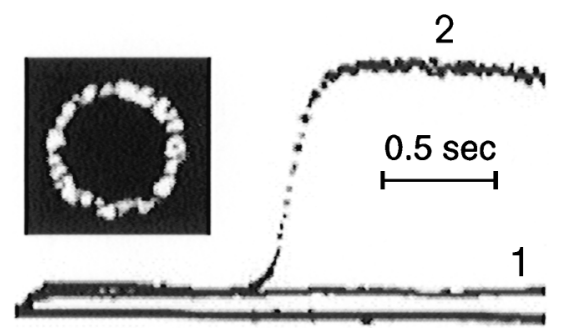

Fig. 3. Time dynamics of the generated radiation.

the amplification of randomly scattered light passed through the filtering system. The intensity distribution in the far field has a speckled ring structure (inset in Fig. 3). Above that threshold the system settles first at a low plateau with a short time scale determined by the photorefractive relaxation time. ${ }^{7}$ Then the system tends to the upper plateau (curve 2) with a slow time scale determined by the supercriticality. At the upper plateau the power of generated light is approximately $30-40 \%$ of the pump wave power.

Here we show that spontaneous hexagon formation results from random small-angle forward scattering of the pump wave inside the crystal. This scattered wave is time independent, and, propagating in forward direction, it does not interact with the pump wave. But after passing through the filtering system it serves as a seed wave for hexagon formation. The intensity of this seed wave is extremely small (approximately $10^{-5 \ldots-6}$ with respect to the pump wave intensity) and determines the threshold of spontaneous hexagon formation.

Let two linearly polarized light waves $F \exp \left(i n_{0} k_{0} z-i \omega_{0} t\right)$ and $B \exp \left(-i n_{0} k_{0} z-i \omega_{0} t\right)$ counterpropagate in a photorefractive crystal. Here $F\left(\mathbf{r}_{\perp}, z, t\right)$ and $B\left(\mathbf{r}_{\perp}, z, t\right)$ are slowly varying complex amplitudes of waves; $k_{0}$ and $\omega_{0}$ are their respective wave numbers and angular frequencies, $n_{0}$ is the refractive index of the crystal, and $\mathbf{r}_{\perp}=(x, y)$ is a transverse coordinate. The length of the crystal along the $z$ axis is equal to $l$ (see Fig. 1). Waves $\mathrm{F}$ and $\mathrm{B}$ can be written as

$$
\begin{aligned}
& F=F_{0}\left[\exp (-\alpha z)+\sum_{\mathbf{k}} \exp \left(i \mathbf{k} \mathbf{r}_{\perp}\right) F_{k}(z, t)\right], \\
& B=F_{0} \sum_{\mathbf{k}} \exp \left(i \mathbf{k} \mathbf{r}_{\perp}\right) B_{k}(z, t),
\end{aligned}
$$

where $F_{0} \exp (-\alpha z)$ is the pump amplitude, $\alpha$ is the linear absorption coefficient of the crystal, $\mathbf{k}=\left(k_{x}, k_{y}\right)$ is the transverse wave vector in the $(x, y)$ plane (here and below we omit the subscript $\perp$ in the wave-vector notation), and $F_{k}$ and $B_{k}$ are weak sideband amplitudes. Space-charge field $E\left(\mathbf{r}_{\perp}, z, t\right)$ can be written as follows:

$$
E\left(\mathbf{r}_{\perp}, z, t\right)=\exp \left(2 i n_{0} k_{0} z\right)
$$

$$
\times \sum_{\mathbf{k}} \exp \left(i \mathbf{k} \mathbf{r}_{\perp}\right) E_{2 k_{0}, k}(z, t)+\text { c.c. }
$$

Then, in a way similar to that described in Ref. 8 and taking into account only terms up to the second order of nonlinearity by amplitudes $B_{k}$ and $E_{2 k_{0}, k}$, we get

$$
\begin{aligned}
\left(\frac{\partial}{\partial z}+i k_{d}+\alpha\right) F_{k} & =i \gamma \sum_{\mathbf{k}_{1}+\mathbf{k}_{2}=\mathbf{k}} E_{2 k_{0}, k_{1}} B_{k_{2}} \\
\left(\frac{\partial}{\partial z}-i k_{d}-\alpha\right) B_{k} & =-i \gamma^{*} \exp (-\alpha z) E_{2 k_{0},-k}^{*} \\
t_{d} \frac{\partial E_{2 k_{0}, k}}{\partial t}= & -\exp (-2 \alpha z) E_{2 k_{0}, k} \\
& +\exp (-\alpha z) B_{k}^{*}
\end{aligned}
$$

where $\gamma \equiv \gamma_{r}+i \gamma_{i}$ is the photorefractive coupling coefficient and $t_{d}$ is the photorefractive relaxation time, which is inversely proportional to the pump intensity $k_{d}=k^{2} / 2 k_{0} n_{0}$. In deriving Eqs. (3) we used the fact that amplitudes $F_{k}$ are quadratic with respect to amplitudes $B_{k}$ and $E_{2 k_{0}, k}$ because the linear terms on the right-hand side of the first equation of system (3) are absent. The result is the splitting of the last two of Eqs. (3) from the first one.

The filtering system selects from the scattered wave a small part that consists of a number of seed beams with transverse wave vectors lying in the Fourier plane on the ring $|\mathbf{k}| \simeq \theta k_{0}$. Thus the boundary condition for the waves $F_{k}$ and $B_{k}$ can be written as

$$
F_{k}(0)=0, \quad B_{k}(l)=F_{k}(l) \exp (i \phi) r+B_{k}^{\text {seed }},
$$

where $B_{k}^{\text {seed }}$ are the amplitudes of the seed beams, $r$ is the amplitude reflection coefficient of the feedback mirror (we also include in this coefficient the losses on the crystal back surface), and $\phi$ is a phase shift of the sideband amplitudes as they propagate from the crystal back surface to the mirror and back. The value of $B_{k}^{\text {seed }}$ is very small in the experiment $\left(\left|B_{k}^{\text {seed }}\right|^{2} \sim 10^{-5 \ldots-6}\right)$.

In a quadratic approximation, which it is natural to term a weakly nonlinear approximation, the intensities of the sidebands are nonzero only on the ring $\left|\mathbf{k}_{\perp}\right| \simeq$ $\theta k_{0}$. Then it follows from the condition of three-wave 
interaction $\mathbf{k}_{1}+\mathbf{k}_{2}=\mathbf{k}$ that the nonlinear terms on the right-hand sides of Eqs. (3) have a nonzero value only for the interaction of those sidebands having traverse wave vectors spaced by angular multiples of $\pi / 3$. Six such vectors form a hexagon. ${ }^{8}$ It is important that in the weakly nonlinear approximation the different hexagons that lie upon the ring do not interact with one another. So in this approximation we can limit the analysis to only one hexagon.

Consider the simplest case, in which the amplitudes $B_{k}^{\text {seed }}, F_{k}, B_{k}$, and $E_{2 k_{0}, k}$ do not depend on $\mathbf{k}$. Then the system of Eqs. (3) reduces in a steady state to

$$
\begin{aligned}
& \left(\frac{\partial}{\partial z}+\alpha+i k_{d}\right) F_{k}=2 i \gamma \exp (\alpha z)\left|B_{k}\right|^{2}, \\
& \left(\frac{\partial}{\partial z}-\alpha-i k_{d}\right) B_{k}=-i \gamma^{*} B_{k} .
\end{aligned}
$$

From Eqs. (5) and (4) we can get the intensity of the weakly nonlinear stationary solution:

$$
\left|B_{k}(l)\right|^{2}=\frac{1-C_{B}^{*}-C_{B} \pm \sqrt{D}}{2|C|^{2}}
$$

where

$$
\begin{aligned}
C & =\frac{2 i \gamma r \exp (i \phi+\alpha l)}{2 \gamma_{i}-i k_{d}-4 \alpha}\left\{\exp \left[\left(2 \gamma_{i}-i k_{d}-4 \alpha\right) l\right]-1\right\}, \\
C_{B} & =C B_{k}^{\text {seed } *}, \quad D=\left(C_{B}+C_{B}^{*}-1\right)^{2}-4\left|C_{B}\right|^{2} .
\end{aligned}
$$

This solution exists provided that $D \geq 0$, from which it follows that the seed intensity $\left|B_{k}^{\text {seed }}\right|^{2}$ must be less the threshold value $\left|B_{k}^{\text {thresh }}\right|^{2}$ given by

$$
\left|B_{k}^{\text {thres }}\right|^{2}=\frac{1}{16|C|^{2} \cos ^{4}(\psi / 2)}, \quad \psi=\arg \left(C_{B}\right) .
$$

The threshold of spontaneous hexagon formation depends on $\psi$ and, as consequence, it depends on a seed phase that cannot be controlled experimentally. Nevertheless, it is natural to expect that among all possible hexagons on the ring the most unstable one will be the hexagon having nearest the zero value of $\psi$. It gives the minimal value of the threshold:

$$
\left|B_{k}^{\text {minthresh }}\right|^{2}=\frac{1}{16|C|^{2}} .
$$

The numerical investigation of the boundary-value problem [Eqs. (3) and (4)] allows us to establish that for $\left|B_{k}^{\text {seed }}\right|^{2} \leq\left|B_{k}^{\text {thresh }}\right|^{2}$ the solution with the positive sign before $\sqrt{D}$ in Eq. (6) is unstable but the solution with the negative sign is stable. Thus if in accordance with the experiment the space-charge amplitude $E_{2 k_{0}, k}$ is zero for $t=0$, the system tends to the stable weakly nonlinear solution [Eq. (6)] that corresponds to the low plateau (curve 1 in Fig. 3). But, if $\left|B_{k}^{\text {seed }}\right|^{2}>\left|B_{k}^{\text {thresh }}\right|^{2}$, spontaneous hexagon formation takes place, and the power tends to the upper plateau (curve 2 in Fig. 3). This upper plateau corresponds to the strong nonlinear solution in which the intensity of the generated wave is of the order of the pump intensity (see, e.g., Ref. 8). Detailed consideration of this solution is beyond the scope of this Letter.

Note that spontaneous hexagon formation in our scheme is essentially a three-dimensional effect. There is no spontaneous generation of any transverse structures in one transverse dimension because in such a case the nonlinear terms in Eqs. (3) would be zero.

In the experiment we determined the threshold of spontaneous hexagon formation by changing the reflection coefficient or by changing $\gamma$. The typical experimental values of system parameters are $\gamma_{r} \simeq 0$, $\gamma_{i} l \simeq 3.2, k_{d} l \simeq \pi, \exp (-2 \alpha l) \simeq 0.54$, and $|r|^{2} \simeq 0.6$. Then it follows from Eq. (9) that $\left|B_{k}^{\text {minthresh }}\right|^{2} \simeq 1.6 \times$ $10^{-6}$, in agreement with experiment.

The authors thank E. A. Kuznetsov and M. Saffman for helpful discussions. Support was provided by the Russian Foundation for Basic Research (grants 97-01-00093, 97-02-18626), the program of government support for leading scientific schools (grant 96-15-96093), the Landau Postdoc Scholarship (KFA, Forschungszentrum, Juelich, Germany), and INTAS (grant 96-0954). P. M. Lushnikov's e-mail address is lushniko@landau.ac.ru.

\section{References}

1. G. Grynberg, Opt. Commun. 66, 321 (1988); J. Pender and L. Hesselink, J. Opt. Soc. Am. B 7, 1361 (1990); R. Macdonald and H. J. Eichler, Opt. Commun. 89, 289 (1992); M. Tamburrini, M. Bonavita, S. Wabnitz, and E. Santamato, Opt. Lett. 18, 855 (1993).

2. T. Honda, Opt. Lett. 18, 598 (1993); T. Honda and P. Banerjee, Opt. Lett. 21, 779 (1996).

3. A. V. Mamaev and M. Saffman, Europhys. Lett. 34, 669 (1996).

4. S. N. Vlasov and E. V. Sheinina, Izv. Vyssh. Ucebn. Zaved. Radiofiz. 26, 20 (1983) [Radiophys. Quantum Electron. 27, 15 (1983)]; G. D'Alessandro and W. J. Firth, Phys. Rev. Lett. 66, 2597 (1991).

5. B. I. Sturman and A. I. Chernykh, Zh. Eksp. Teor. Fiz. 111, 1611 (1997) [JETP 84, 881 (1997)].

6. P. M. Lushnikov, P. Lodahl, and M. Saffman, Opt. Lett. 23, 1650 (1998).

7. B. Sturman and V. Fridkin, The Photovoltaic and Photorefractive Effects in Noncentrosymmetric Materials (Gordon \& Breach, New York, 1992).

8. P. M. Lushnikov, Zh. Eksp. Teor. Fiz. 113, 1122 (1998) [JETP 86, 614 (1998)]. 